\title{
Toxic Megacolon due to Fulminant Amebic Colitis in a non Endemic Area
}

\author{
Sarah Hugelshofera, d, David Petermann ${ }^{\mathrm{b}}$, Christina Orasch ${ }^{\mathrm{c}}$, Lucas Liaudet ${ }^{\mathrm{a}}$
}

\begin{abstract}
We report a case of fulminant necrotizing colitis and toxic megacolon in a middle-aged woman, due to infection with Entamoeba histolytica acquired in a non endemic zone of amebiasis, and requiring emergent total colectomy due to the occurrence of colon perforation and peritonitis. Fulminant colitis is a rare complication of amebiasis, which has been only exceptionally reported in the absence of a recent travel in endemic areas. In the case presented herein, we discuss the possible role of the use of large doses of loperamide, as well as that of a co-infection with Norovirus, in triggering the fulminant course of the disease.
\end{abstract}

Keywords: Amebic colitis; Toxic megacolon; Entamoeba histolytica; Norovirus; Loperamide

\section{Introduction}

Amebiasis is a parasitic infection caused by the ingestion of the protozoan Entamoeba histolytica via contaminated food or water or through fecal-oral contact. Its prevalence is high in India, Africa, Mexico, parts of Central and South America, and it may occasionally be diagnosed in non endemic areas, in migrants and travelers returning from endemic regions. Amebiasis is asymptomatic in up to $90 \%$ of infected per-

Manuscript accepted for publication September 24, 2012

\footnotetext{
${ }^{a}$ Department of Intensive Care Medicine, University Hospital Medical Center and Faculty of Biology and Medicine, Lausanne 1010, Switzerland

${ }^{\mathrm{b}}$ Department of Visceral Surgery, University Hospital Medical Center and Faculty of Biology and Medicine, Lausanne 1010, Switzerland ${ }^{\mathrm{c}}$ Service of Infectious Diseases, University Hospital Medical Center and Faculty of Biology and Medicine, Lausanne 1010, Switzerland ${ }^{\mathrm{d}}$ Corresponding author: Sarah Hugelshofer, Department of Critical Care Medicine and Burn Center, Lausanne-1010, Switzerland.

Email: sarah.hugelshofer@chuv.ch
}

doi: http://dx.doi.org/10.4021/jmc900w sons, in the others it presents as diarrhea (amebic dysentery) or extraintestinal disease (mainly hepatic abscesses) [1,2]. Rarely ( $<3 \%$ of cases), the disease takes a fulminant course characterized by necrotizing colitis that may lead to toxic megacolon with a mortality rate of more than $40 \%$ [3]. We present a patient from a non endemic zone developing such a major complication of amebiasis.

\section{Case Report}

A 45-year-old woman of West African origin was admitted to our hospital after a 2 weeks history of progressive abdominal cramps and bloody diarrhea. She had not travelled to her country nor left Western Europe for 5 years, but she reported consumption of imported African vegetables ("gumbo") purchased in a local grocery store 3 days before symptom onset. Her past history was unremarkable. She was treated by her family physician with loperamide and ciprofloxacine for 5 days without improvement, and was then admitted to a secondary care hospital. A stool specimen was taken and showed a positive PCR for Norovirus. The patient was treated by supportive care, but after 3 days, she was transferred to our tertiary care hospital due to progressive hypotension and increasing abdominal pain. On admission, the patient was in a poor general condition, her core temperature was $38.3{ }^{\circ} \mathrm{C}$, arterial blood pressure was $100 / 50 \mathrm{mmHg}$ and heart rate was $100 \mathrm{bpm}$. The abdomen was dilated, with diffuse tenderness and signs of peritonitis. Laboratory examination showed normal leukocyte count at $6.2 \mathrm{G} / \mathrm{L}$, a $\mathrm{C}$ reactive protein at $222 \mathrm{mg} / \mathrm{L}$, signs of acute renal failure (creatinine $162 \mu \mathrm{mol} / \mathrm{L}$ ) and disseminated intravascular coagulation. HIV serology was negative. An abdominal CT scan revealed large bowel dilatation $(8 \mathrm{~cm})$ with a thickened colonic wall and a peri-colic fat infiltration (Fig. 1A). While the patient was prepared for emergency surgery, she developed cardiac arrest due to ventricular fibrillation requiring cardio-pulmonary resuscitation that was successful after 10 minutes. An emergent total colectomy was then performed for necrotic pancolitis with perforation (Fig. 1B) and the patient postoperatively was admitted to the intensive care unit (ICU). Histopathological examination revealed extensive large bowel 

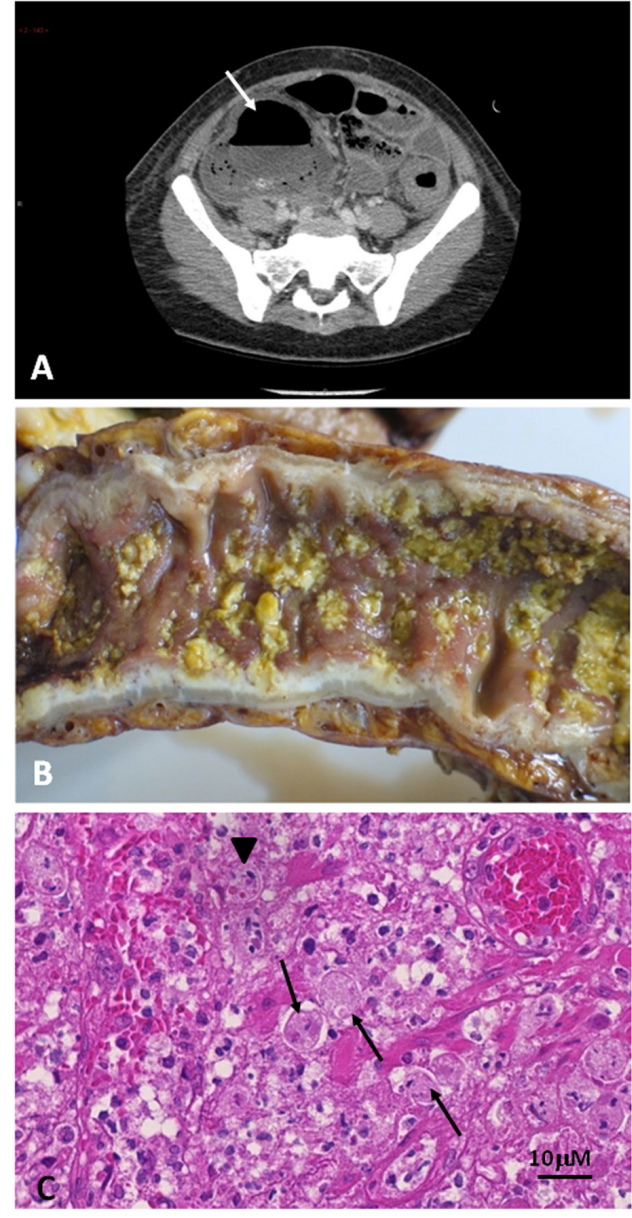

Figure 1. Acute necrotizing colitis due to invasive amebiasis. A. Marked dilatation of the colon lumen with thickened colonic wall, particularly at the level of the caecum (arrow). B. Colectomy specimen showing diffuse necrosis of the colon. C. Histopathological study of the colon, identifying numerous amebic trophozoites (arrows), with overt pictures of hemophagocytosis (arrowhead).

necrosis with multiple deep ulcers, massive infiltration with Entamoeba trophozoites and signs of hemophagocytosis (Fig. 1C). The patient was successfully treated with a ten day course of metronidazole followed by 7 days of paromomycin for eradication of the documented intraluminal cysts. She was transferred to the general ward after a 3 weeks' stay at the ICU and then to a neurological rehabilitation unit for the management of moderate post-anoxic encephalopathy.

\section{Discussion}

Fulminant amebic colitis is an uncommon and severe complication of infection with E. histolytica. Toxic dilatation is rare and portends a poor prognosis. The diagnosis is often delayed and is generally made histologically in operative or postmortem specimens. Risk factors for invasive disease include the virulence of the strain of E. histolytica, as well as host factors including genetic susceptibility, young age, pregnancy and impaired immunity (for example. due to treatment with corticosteroids, malignancy, malnutrition, alcoholism, and HIV infection) [4-6]. The patient under discussion developed fulminant colitis within a non endemic region, a feature which has only been rarely reported in the absence of recent travelling [7]. Considering her African origin, she could have been infected prior to her arrival in Europe, which would be consistent with a long-lasting (more than 5 years) carrier state. An alternative possibility would be a recent contamination due to the ingestion of vegetables obtained in a local African grocery. In such a scenario, the vegetable might have either carried amebic cysts imported from Africa (given their ability to survive several weeks in soil or water) or have been locally contaminated by the African food seller. In either case, two potentially precipitating factors may have been the co-infection with Norovirus and treatment with large amounts of loperamide. Considering the former, it is noteworthy that there is no previous report of co-infection with Norovirus and E. histolytica. With respect to the latter, several reports have suggested that loperamide may promote toxic intestinal dilatation in case of gut infections with invasive or toxin-producing organisms [8]. Indeed, loperamide inhibits intestinal smooth muscle function, thereby prolonging the contact of invasive organisms (such as $E$. histolytica) with the mucosa. It is also particularly noteworthy that the patient developed an extremely severe form of the disease in the absence of impaired immune status, which further supports the potential role played by the coinfection with Norovirus and the treatment with loperamide.

We conclude that amebiasis may occur in non endemic zones and may be complicated by acute fulminant necrotizing colitis and toxic megacolon, requiring emergency resection surgery combined with antiamebic treatment. The use of loperamide may represent a major precipitating factor for such a complication, and should be avoided when signs of mucosal involvement, such as rectal bleeding and fever, are present during the course of gastroenteritis. This is the first case report of co-infection with Norovirus and E. histolytica, which may have contributed to the particularly severe clinical course.

\section{Grants}

LL is supported by the Swiss National Fund for Scientific Research (grant Nr 310030_135394/1.

\section{Conflict of Interest Disclosure}

The authors have no conflict of interest to disclose with re- 
spect to this article.

\section{References}

1. Haque R, Huston CD, Hughes M, Houpt E, Petri WA, Jr. Amebiasis. N Engl J Med. 2003;348(16):1565-1573.

2. Weinke T, Friedrich-Janicke B, Hopp P, Janitschke K. Prevalence and clinical importance of Entamoeba histolytica in two high-risk groups: travelers returning from the tropics and male homosexuals. J Infect Dis. 1990;161(5):1029-1031.

3. Aristizabal H, Acevedo J, Botero M. Fulminant amebic colitis. World J Surg. 1991;15(2):216-221.

4. Arora A, Sandip S, Mukund A, Patidar Y. Fulminant nec- rotizing amoebic colitis. Travel Med Infect Dis. 2012.

5. Ishioka H, Umezawa M, Hatakeyama S. Fulminant amebic colitis in an HIV-infected homosexual man. Intern Med. 2011;50(22):2851-2854.

6. Ximenez C, Moran P, Rojas L, Valadez A, Gomez A. Reassessment of the epidemiology of amebiasis: state of the art. Infect Genet Evol. 2009;9(6):1023-1032.

7. Shimada S, Mizumoto T, Nishioka R, Fukami K, Kuramoto M, Nomura K, Aoki N, et al. Acute fulminant necrotizing colitis caused by amebiasis: report of a case. Surg Today. 2002;32(8):738-741.

8. McGregor A, Brown M, Thway K, Wright SG. Fulminant amoebic colitis following loperamide use. J Travel Med. 2007;14(1):61-62. 